\title{
The NHS Information Management and Technology strategy from a mental health perspective
}

\section{Susan Knight}

Healthcare organisations are currently in the midst of a period of fundamental change. Mental health services in particular have moved away from institutional care towards a complex inter-related community health social and voluntary model of service delivery, involving a diverse range of care professionals and commissioners.

Against this background, where different aspects of patient care are delivered from very different sources, the need for swift access to comprehensive information, and communication between groups involved in treatment, is critical if the patient is to receive relevant beneficial treatment.

Computerised information offers clinicians an exceptional opportunity to improve standards of patient care not only by accessing and exchanging relevant information on individual patients, but also by immediate access to pertinent and up-todate research in their field. Rapid developments in computing technology can offer healthcare professionals new opportunities for achieving their objectives.

Clinical and management information systems have considerable potential for improving the efficiency of care delivery and communication, and thus influencing the quality of service and outcomes. This paper will enable psychiatrists to be aware of some of the key issues which must be addressed for successful information management and technology strategies in mental healthcare.

\section{The National Information Management \&Technology strategy}

The overall aim of the Information Management and Technology strategy (IM \& T) is to support better care and communication through the appropriate use of information management and technology in the NHS. There are a number of key components within this overall aim which are presently being developed by the NHS in England. All are needed to support the care of individuals and some to enable more effective communication with many other non-clinical aspects of the organisation (NHSE, 1992).

IM \& $\mathrm{T}$ used in the provision of clinical care can now integrate health information on identified individuals through the use of electronic systems based on those individual patients. These patient based systems, if networked, can be accessed via a personal computer in multiple locations, thus providing the clinician with rapid access to relevant information in the patient health record. Access to patient records is on a 'need to know' basis safeguarded by stringent confidentiality and security measures. Due to the highly sensitive nature of patient health records, practitioner and patient have concerns over security and confidentiality issues. These concerns about computerised patient data have been addressed by additional new rules and regulations.

\section{Security and confidentiality}

The Data Protection Act (HMSO, 1984) covers personal data stored on computers in the UK. Local initiatives such as directives on changing passwords regularly, taking action against employees for breach of privacy, physical security of data stores and systems terminals together with Organisation for Economic Cooperation and Development (OEDC) guidelines and European Community (EC) mandates have a major impact on the management of healthcare information (HMSO, 1993).

Susan Knight, MA, is a qualified psychiatric social worker. She has successfully implemented an information system in an acute hospital for Huddersfield NHS Trust. She is on a secondment to the IMG and is presently responsible for the Enabling IM \& T Support for Care in the Community and the Mental Health Minimum Data Set projects. She can be contacted at Information Management Group, Room 5W 12, Quarry House, Quarry Hill, Leeds LS2 7UE. 
To support and underpin the IM\&T strategy, a security framework has been developed to enable good security practice to be introduced for NHS information systems. It consists of five key elements:

(i) The development and implementation of a security policy within each organisation.

(ii) The appointment of an individual to oversee the implementation of the policy and associated mechanisms and procedures.

(iii) The review of the organisations' legal obligations in relation to the Data Protection Act.

(iv) The review and implementation of relevant baseline security measures as outlined in Basic Information System Security, published in 1992.

(v) The implementation, where appropriate, of security measures to meet the requirements of NHS-wide networking.

The framework is designed to introduce a level of security into information systems, in line with the requirement of the clinical professions, the patients and NHS management. The key elements of the framework are recognised and accepted by information security specialists worldwide from all spheres of operation.

Responsibility for the safe-keeping of NHS information (particularly personal health information), rests with the owners or custodians of that information. The consequences of a breach of confidentiality or of any other aspect of security will rest with the management of the organisation which the owners or custodians belong to. The cost to an NHS organisation of losing or revealing information through a lack of properly managed security is very high, not only because of the legal considerations but also because of the embarrassment and loss of the confidence of patients and professionals.

The success of the IM \& T strategy depends upon the confidence of the patient, the clinicians and management in the security of information held on NHS systems and transferred across networks. To gain such confidence, all IM \& T specialists must address the requirements of the security framework in their locality (NHSE, 1994c; Audit Commission, 1995).

\section{The four strategic initiatives}

\section{Facilitating progress through projects}

The Information Management Group (IMG) has over the last few years sponsored a number of projects which aim to demonstrate the use of $I M \& T$ in key areas of clinical care and service management. One of these has been the Community Information Systems for Providers (CISP) project. This three year programme, now complete, involved sites throughout the UK and demonstrated the use of IM \& $T$ in meeting the information needs required to support the Caring for People initiative. The CISP project concluded that Community Information Systems must be:

Person-based, so that the healthcare record is held for each individual which can then be referenced to the individual's NHS number.

Integrated, so that duplicated data entry is minimised and information is available in other designated NHS systems.

Operational, aimed at supporting clinical work so that additional efforts required to capture information specifically for management purposes is minimised.

Secure, so that information is only made available on a 'need to know' basis to authorised staff.

One of the achievements of the CISP project was the agreement of the inadequacy of Korner data sets for purchasing purposes. Work was undertaken in respect of Health Benefit Groups and Care Aims in a Community Contracting Minimum Data Set (CCMDS). Work on the mental health minimum data set is currently underway and is being piloted at nine sites throughout England. The CISP message for the necessity of integrated personbased computer systems containing clinically relevant information in community focused care, has been widely accepted and will undoubtedly improve communications within and between organisations.

\section{Enabling people}

One of the greatest challenges to the successful introduction and implementation of IM \& $\mathrm{T}$ in the NHS, is the development of an information culture. This can only be achieved through extensive programmes of awareness, training and education that will allow clinicians and managers to fully appreciate the benefits of a patient based information system. The IMG finances such programmes in partnership with the Royal Colleges and other professional bodies. It is essential for Trusts to ensure that all staff are equipped to use information systems productively and are able to use the information that they get from such systems. Lack of skills and knowledge are frequently compounded by negative attitudes towards information systems; whether an information system is a success or not depends on the attitude of the staff who are using it. Even the best system will fail unless staff are committed to it. 
Value for money

The NHS Executive has issued guidance on the preparation of a sound business case for any investment in $\mathrm{IM} \& \mathrm{~T}$ to ensure maximum benefit is gained from the expenditure (NHSE, 1995c). Compliance with these standards in the long term, reduces costs and risks for both local organisations and the NHS as a whole. Particularly in community systems, inter-connectivity is extremely important as services are provided from bases in a wide geographical area. Corporate standards must therefore be adopted and adhered to, otherwise inter-connection will rapidly become unaffordable, if not impossible.

Within the NHS there is the difficulty that many purchasers of systems have never gone through such procurement before and may often have insufficient knowledge about the type of systems on the market. The preparation of a business case agreed by both managerial and clinical staff should ensure that all members of the team know what they're getting. Adequate consultation with clinical staff is essential.

\section{Developing an infrastructure}

Realising the aims of the IM \& T strategy requires more efficient communications and information processing within organisations which in turn requires a common infrastructure. The NHS Executive has determined the shape of this infrastructure and in cooperation with the service expects to have it largely in place by 1997 (Information and Management Group of the NHSE, 1994).

\section{Major factors in the infrastructure}

National comparisons show that there are many ways in which health services can be delivered to treat illness and promote health but there is a common factor, the needs of the individual receiving the service. The term 'seamless care' may seem somewhat overused but it does capture the element of this requirement. In mental health care particularly, the individual moves in and out of the service and receives input from both primary and secondary care, the Local Authority, both Housing and Social Services and voluntary organisations. This presents difficulties in planning an effective total care package and in tracking what services have been received by the patient. Providing information that is focused on the person, rather than on the organisation or professional group, marks the fundamental shift from the activity based systems that many community organisations presently use.

The actual use of a computerised information system in a depot clinic may help to illustrate these points. In the Mental Health Directorate of Huddersfield NHS Trust, we introduced a system to capture information regarding patients attending for depot injections. This included PCs in various clinics and the out-patient department and handheld barcoding devices. The problem in the past had been that details of patients had been kept on cards in the out-patient department of the hospital. Some patients would come to the hospital for their injections. Staff also held depot clinics at various other locations throughout the Trust, as well as community psychiatric nurses (CPNs) giving people injections in their own homes. It proved extremely difficult to keep track of who had given the medication to the patient, what dose and when, particularly when the cards were taken from the out-patient clinic by the CPNs to the various locations.

In consultation with the nursing and medical staff concerned, a computer based system was developed whereby the nurse giving the injection could transfer all the patient's demographic details from the hospital's master patient index, using either the patient's name or hospital number. They then used a barcoding device to record the type and dosage of medication given, the site of the injection, the date, the time, the place and the member of staff involved. They could also record side-effects. The system would produce appropriate appointment letters and would also flag up those patients who had not attended on the given day, together with some indication of how urgent it was to contact each of these individual patients. The medical staff could also change the medication, dosage and duration using the barcoding device.

This fairly straightforward information system revolutionised the information that was now available on patients attending for depot injections. Communications were hugely improved and the service given to the patients was of a far higher quality than before, in so much as there were no missing or lost cards and patients who needed following up were quickly notified. All staff involved, even those sceptical at the beginning, were extremely enthusiastic about the system, as it provided almost immediate benefits to all concerned.

Systems such as these are a change from the bias that there has been in the NHS towards administrative and financial systems. Such systems were 
introduced presumably because managers control budgets and wanted reliable information on which to manage. They have frequently failed to achieve their goal because the systems have not been fed by reliable data used for operational clinical purposes.

\section{Replacement NHS number}

The key to effective sharing of information about patients in NHS information systems, while maintaining privacy is the ability to identify each person in an unambiguous and unique way which is common throughout the NHS. It has been decided that the NHS number should be used as a unique personal identifier. The present NHS number is not suitable for use in information systems as it has 22 different formats which are difficult to validate. Registrars of births will commence using new format NHS numbers from December 1995. Starting in January 1996, the NHS number replacement programme will introduce the new format 10 digit number which includes a check digit.

\section{NHS administrative registers}

Many NHS organisations need to have administrative details about the populations they serve. Population registers such as the FHSA patient register are not designed for sharing and have limitations for other users. The NHS Executive has commissioned software for population databases called NHS Administrative Registers (NHSARs) which are suitable for sharing. This register is a database which holds a common core of administrative data (people, places, organisations and links) covering all individuals living within a natural community. It is an independent organisation which can combine and separate data to form new geographical boundaries. It has interfaces to purchaser and provider systems and exchanges data electronically, to all participating organisations, subject to stringent security and confidentiality safeguards. It has a core security policy which has been approved by the Data Protection Registrar.

\section{Read codes}

The Read coded clinical terms are a set of nationally agreed terms and synonyms, developed by and for clinicians, as used in current clinical records. The terms are coded for use in person- based computerised systems and provide clinical audit, research and decision support. These are essential if comprehensive data about clinical encounters is to be captured in computers in all healthcare environments. Without capturing such data, the electronic patient record is impossible and there will be a severely restricted source of data for audit, outcomes, care protocols, management of resources and contracting. Cross references to classifications such as ICD-10 and OPCS -4 allow for statistical analysis of accurate and timely data gathered using the terms. The clinical terms are a fully comprehensive coded thesaurus of coded clinical terms, synonyms, abbreviations and qualifiers. A recent project has successfully expanded the Read codes to become an agreed thesaurus of clinical terms, synonyms and abbreviations for the medical profession. The project involved establishing 43 speciality working groups representing every medical speciality including mental health. A total of over 2000 clinicians and 55 working groups have been involved in these projects, coordinated by the NHS Centre for Coding and Classification (NHS Executive, 1995b).

\section{NHS-wide networking}

The NHS-wide networking system is an initiative at the heart of the IM \& T strategy for the NHS, it is an integrated approach to inter-organisational communication, which includes purchasers, providers and GPs within the NHS. The aim is to support and improve the flow of information on which patient care depends. NHS-wide networking covers everything from mobile communications to the electronic transmission of images. It focuses on communications between organisations, rather than within an individual organisation. There are four main elements to the NHS-wide networking programme which seek to:

(i) Rationalise NHS voice communication facilities. Negotiation and implementation of bulk telephone discounts from British Telecom and Mercury will give savings in excess of $£ 10 \mathrm{~m}$ per year nationally, with almost all parts of the NHS benefiting.

(ii) Set up a national network for the electronic transmission of data between computers, organisations and people throughout the NHS.

(iii)Improve NHS radio communications, particularly those used by ambulance services.

(iv)Establish an integrated management organisation to oversee all types of networking, data, voice, images and radio. 


\section{Integrated clinical workstation}

Despite considerable efforts over the past 20 years, patients' records today are still predominantly paper based. Current trends in service organisations, a more mobile population, a growing number of elderly people, people with mental illness being predominantly cared for in the community and more demanding expectations of the NHS, point to the need for systems which support healthcare professionals in sharing information and coordinating their activities for the benefit of the patient. Recent White Papers, contractual obligations and the requirements for clinical audit reinforce this. Clinical systems that hold electronic patient records are now becoming available. To date, effective developments have been in primary care.

The integrated clinical workstation programme has been established to take the issue forward. The aim is to provide doctors, nurses and the professionals allied to medicine, with quick and easy access to the information they need to care for patients when and where they need it, and in a form they find most useful. This information could include x-rays and other images, manuscript notes, letters, test results, guidelines, protocols and research information.

A key requirement is a straightforward interface that healthcare professionals do not need extensive training to use. This is one of the principal aims of the programme. Workstations will take various forms depending on who is using them and where, they may be desk based or portable, they may use light pens, barcode readers and voice and handwriting recognition systems. To be fully effective, integrated clinical workstations will require access to:

(i) An accurate up-to-date master index of all patients linked to the planned national NHS administrative register

(ii) A range of local and remote information systems which holds clinical and administrative information about individual patients

(iii) A scheduling service which can handle outpatients, in-patients, therapies and all kinds of patient appointments

(iv) Communication facilities between individual healthcare practitioners.

A longer term research and development project is the electronic patient record in a hospital context, and current work on this should be providing results by 1997 . In the foreseeable future, it is anticipated the use of workstations will be able to capture and retrieve multi-media health data.

\section{Healthcare Resource Groups}

Health care resource groups (HRGs) are casemix groups which have been developed to group data on the basis of both clinical and resource homogeneity. Casemix groups, particularly HRGs, are used to make management decisions on service provision. Because these decisions have an impact on clinicians, the National Casemix Office (NCMO) facilitated the development of the groups by using clinical working groups - committees of clinicians from particular specialities with leaders nominated by their relevant medical professional associations. Work has taken place in developing HRGs in mental health, mainly assessing costings for inpatient episodes with a limited amount of community based work. This will be extended during 19951996 by the IMG.

\section{Future work}

Mental health services are a key target for the IMG for the next 2-3 years. A recent survey (Glover, 1994, personal communication) showed a paucity of person-based information systems within mental health services. The Generic Statement of Need (NHS Executive, 1995c) for mental health systems has been commissioned and is available free of charge. Also available is Describing Mental Health Services (NHS Executive, 1995a), which brings together all relevant present initiatives in $\mathrm{IM} \& \mathrm{~T}$ in this area.

\section{A minimum data set for mental health}

The Department of Health and the NHS Executive are reviewing the information that is currently available to support clinical care and the provision, management and purchasing of mental health services. A user group has been established to advise on this review and includes clinicians and managers from mental health service purchasers and providers as well as social services.

The new proposals will change the main focus of information from administration to patient care. Information will be recorded about patients care 
plans when clinical reviews occur. This can happen for in-patients, day-patients and out-patients alike.

A minimum data set is a set of items of information which all providers of any type of service in the NHS would need to collect to do their work. By introducing a standardised structure and set of definitions, the Department of Health ensures that this information can be aggregated and comparisons can be made reliably. A clinical minimum data set thus represents the core information about individual patients that is necessary to support clinicians working within multi-disciplinary teams in the delivery of care to their patients. National statistics will be derived directly from these care plans, local contracts and budgets will also be set on the basis of this information.

An awareness pack on this new data set has recently been sent out to all clinical directors of mental health services, directors of public health, directors of social services, voluntary organisations and user groups.

\section{Enabling IM \& T support for community focused care}

Three broad areas of work have been defined to take place over the next two years, using advisory panels comprising healthcare professionals, information specialists and managers from local NHS organisations, social services professionals, NHS Executive and Department of Health staff and research groups.

The development and use of care packages

The development of care packages to support the delivery, planning and commissioning of community healthcare services, in particular mental health services. Work includes:

(i) The requirements for a common shared language which underpins the development of care packages, and other classifications of community based healthcare, which is accepted and understood by healthcare professionals and can be consistently applied.

(ii) The development of classifications of mental healthcare treatments and their relationship to and use in the development of care packages.

(iii) The inclusion and monitoring of appropriate outcome measures in care packages for the mentally ill. (iv) The business case that supports the use of a care package approach.

(v) The requirement for national guidance and training materials.

IM \& $\mathrm{T}$ developments in supporting community care

Supporting the delivery, planning and commissioning of health and social care for clients or patients within a community setting including:

(i) The requirements for a common set of definitions and terms, shared by health care professionals and organisations and social care professionals and organisation, for use in the planning and delivery of integrated care.

(ii) The development of client classifications, and resource input or treatment classifications to support strategic planning and resource allocation, operational planning and delivery of services and audit.

(iii) A review of existing practices and procedures for collecting, sharing and using client based information.

(iv) The development of mechanisms for sharing experiences and good practice in the joint use of information and systems by health care organisations and social services.

(v) IM\&T support for providing local populations with consistent information on the availability of local community care services.

Work will focus principally on older people, those with mental health and learning disabilities and their service requirements.

IM \& $T$ for practice focused healthcare

Supporting the IM \& T needs of primary healthcare teams including:

(i) A fuller understanding of the current status of IM \& T support for primary healthcare teams (PHCTs).

(ii) Consolidating previous work on the information needs of PHCTs and approaches to meeting these.

(iii) Defining the requirements for shared, consistent information to support integrated child health services.

The idea is to build on good practice in these areas already taking place within the NHS. I would be pleased to hear from any clinician whose organisation is interested in taking part in this work. 


\section{Conclusions}

To date, there is an intrinsic lack of interest in information systems among many clinical staff. Clinicians see their role as limited to the clinical care of patients and reject the notion that they have a wider role in managing information and the business of the Trust. This disinterest will continue until clinicians see benefits arriving from information and understand its value and potential. This means that information systems must be made more clinically appropriate, timely, accurate and useable. Information needs to be actively managed for all practical purposes, this means it has to be computerised but computers, information and technology must not be allowed to drive the process of information management, only to serve it. It is vital never to lose sight of what the information is needed for and how Trusts use it.

I hope that some of the potential benefits will be appreciated and mental health services take an active part in the exciting new developments that technology of the 1990s has to offer.

\section{References}

Audit Committee (1995) For your Information. A Study of Information Management and Systems in the Acute Hospital. London: HMSO.

HMSO (1984) Data Protection Act. London: HMSO.

- (1990a) Access to Medical Records Act. London: HMSO

- (1990b) Computer Misuse Act. London: HMSO.

- (1993) Privacy Act 1993. London: HMSO.

Information Management Group of the NHS Executive (1994) Implementing the infrastructure. A Handbook for IMET Specialists. Department of Health.

National Health Service Executive (1992) An Information and Technology Strategy for the NHS in England. Department of Health, HMSO.

- (1994a) Clinical Coding Support Unit, Data Quality, Patient Classification Systems and Audit. A Recent Study. Proceedings of Healthcare 1994 conference. Department of Health.

- (1994b) Clinical Workstations: Focus for the Future. Department of Health.

-(1994c) Draft guidance for the NHS on Confidentiality, Use and Disclosure of Personal Health Information. Department of Health.

- (1995a) Describing Mental Health.

- (1995b) Read Codes and the Terms Project: a Brief Guide. February 1995.

- (1995c) Generic Statement of Need for a Mental Health Information System.

The Welsh Office (1994) Draft guidance for the NHS on Confidentiality Use and Disclosure of Personal Health Information. HMSO.

\section{Forthcoming CPD workshops}

\section{Assertive Case Management}

Thursday 23 November 1995, London
ECT Training Day

Monday 11 December 1995, Dublin

For further details please contact Annabel Thomas, CPD Department, Royal College of Psychiatrists, Tel. 01712352351 ext.145; fax 01712596507 